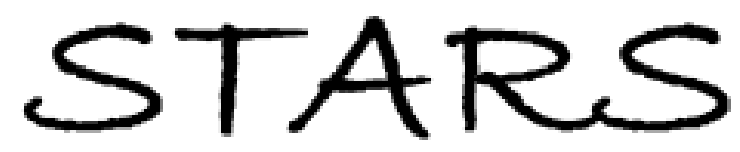

University of Central Florida

STARS

$1-1-2003$

\title{
Discrete vector solitons in two-dimensional nonlinear waveguide arrays: Solutions, stability, and dynamics
}

\author{
J. Hudock \\ University of Central Florida \\ P. G. Kevrekidis \\ B. A. Malomed \\ Demetrios N. Christodoulides \\ University of Central Florida
}

Find similar works at: https://stars.library.ucf.edu/facultybib2000

University of Central Florida Libraries http://library.ucf.edu

This Article is brought to you for free and open access by the Faculty Bibliography at STARS. It has been accepted for inclusion in Faculty Bibliography 2000 s by an authorized administrator of STARS. For more information, please contactSTARS@ucf.edu.

\section{Recommended Citation}

Hudock, J.; Kevrekidis, P. G.; Malomed, B. A.; and Christodoulides, Demetrios N., "Discrete vector solitons in two-dimensional nonlinear waveguide arrays: Solutions, stability, and dynamics" (2003). Faculty Bibliography 2000s. 3822.

https://stars.library.ucf.edu/facultybib2000/3822

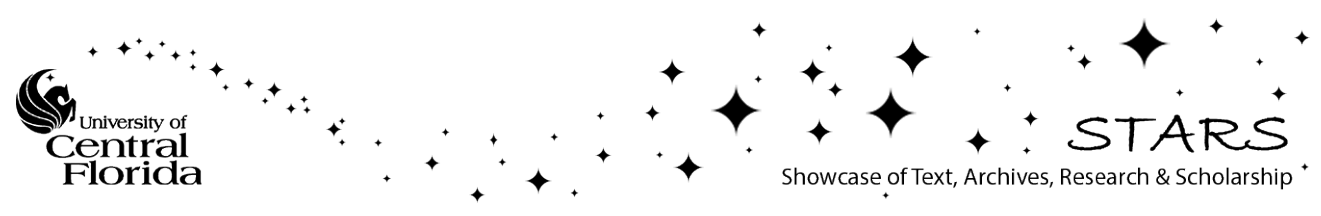




\title{
Discrete vector solitons in two-dimensional nonlinear waveguide arrays: Solutions, stability, and dynamics
}

\author{
J. Hudock, ${ }^{1,2}$ P. G. Kevrekidis, ${ }^{1,3}$ B. A. Malomed, ${ }^{4}$ and D. N. Christodoulides ${ }^{2}$ \\ ${ }^{1}$ Theoretical Division and Center for Nonlinear Studies, Los Alamos National Laboratory, Los Alamos, New Mexico 87545 \\ ${ }^{2}$ School of Optics/CREOL, University of Central Florida, Orlando, Florida 32816 \\ ${ }^{3}$ Department of Mathematics and Statistics, University of Massachusetts, Amherst, Massachusetts 01003-4515 \\ ${ }^{4}$ Department of Interdisciplinary Studies, Faculty of Engineering, Tel Aviv University, Tel Aviv 69978, Israel
}

(Received 21 December 2001; revised manuscript received 23 January 2003; published 21 May 2003)

\begin{abstract}
We identify and investigate bimodal (vector) solitons in models of square-lattice arrays of nonlinear optical waveguides. These vector self-localized states are, in fact, self-induced channels in a nonlinear photonic-crystal matrix. Such two-dimensional discrete vector solitons are possible in waveguide arrays in which each element carries two light beams that are either orthogonally polarized or have different carrier wavelengths. Estimates of the physical parameters necessary to support such soliton solutions in waveguide arrays are given. Using Newton relaxation methods, we obtain stationary vector-soliton solutions, and examine their stability through the computation of linearized eigenvalues for small perturbations. Our results may also be applicable to other systems such as two-component Bose-Einstein condensates trapped in a two-dimensional optical lattice.
\end{abstract}

DOI: 10.1103/PhysRevE.67.056618

\section{INTRODUCTION}

Nonlinear lattice equations naturally appear in the description of various physical systems. A paradigmatic example of a lattice dynamical model is the discrete nonlinear Schrödinger (DNLS) equation with the cubic on-site nonlinearity [1]. This equation has important applications in biophysics [2], nonlinear optics [3], solid-state physics [4], and Bose-Einstein condensates [5]. In experimental settings, the DNLS model finds its most straightforward realization in nonlinear optics, in terms of linearly coupled nonlinearwaveguide arrays, as it was first proposed in Ref. [3] and implemented experimentally in Refs. [6,7] (a systematic presentation of the experimental results is given in a recent work [8]). In this system, self-localized excitations (discrete solitons) are possible as a result of the interplay between the Kerr nonlinearity and discrete linear coupling. Many properties of optical discrete spatial solitons have been systematically explored in theory and experiment, including generalizations to diffraction management $[9,10]$, diffractionmanaged solitons [11], and soliton transport [12].

The DNLS equation for two-dimensional (2D) nonlinear lattices has also been investigated (see, e.g., Refs. $[13,14]$, and references therein). In this case, the discreteness has been shown to substantially modify the dynamics $[15,16]$. As a result, unstable broad discrete solitons in 2D waveguide arrays with a self-focusing cubic (Kerr) nonlinearity tend to quasicollapse into stable narrow localized modes [15]. Spatiotemporal compression is also possible in these systems, as it was demonstrated theoretically [17] and experimentally [18].

In a very recent experiment, two-dimensional discrete solitons were observed for the first time in photorefractive crystals [19]. In this case, the 2D waveguide array was optically induced by interfering pairs of plane waves in highly anisotropic (in terms of nonlinearity) photorefractive crystals [20]. In addition to this approach, photonic-crystal fibers (PCFs) may provide an alternative avenue where such 2D discrete self-trapped states can be observed.
PACS number(s): 41.20.Jb, 63.20.Pw
Another physical setting where 2D discrete vector solitons can appear happens to be two-component Bose-Einstein condensates (BECs) trapped in a two-dimensional optical lattice. In this case, two DNLS equations coupled by nonlinear cross-phase modulation (XPM) and linear terms can also be applied to the description of the BEC dynamics in the usual mean-field approximation. First of all, a system of two Gross-Pitaevskii (GP) equations with exactly the same couplings as in the case of the system of two coupled optical NLS equations directly describes a binary condensate in the form of a mixture with two different hyperfine states of one species of atoms [21]. Nonlinear interaction between the components is generated by atomic collisions, while linear coupling may be readily induced by an external microwave or radio-frequency field that induces Rabi [21] or Josephson [22] oscillations between populations of the two states. Then, if a BEC is placed in a 2D optical lattice [23], its dynamics will be adequately described by the lattice version of the GP equations.

A problem that may be promising for the experimental realization, and is of considerable theoretical interest in its own right, is to analyze the formation and the stability of $2 \mathrm{D}$ vector discrete solitons in the $2 \mathrm{D}$ bimodal DNLS model. This is the subject of the present work. It is necessary to mention that strongly localized vector (two-component) discrete solitons have been identified in models of 1D nonlinear waveguide arrays, where two fields interact through XPM [24,25]. Like their continuous counterparts [26-30], these vector solitons may have components of different types (bright, dark, or antidark). In particular, symbiotic bright-dark and dark-antidark pairs were predicted in such systems $[24,25]$.

In the model considered below (with the nonlinear and/or linear couplings between the two modes), vector discrete solitons, as stationary solutions to the coupled equations, are obtained using numerical Newton-type methods, and the parameter regions where these solitons are stable or unstable are found. The eigenvalues responsible for the instability of the solitons are identified, and in cases where solitons are unstable, their evolution is directly simulated. 
The rest of the paper is organized as follows. The models are formulated in Sec. II, where we also consider their linear spectrum and outline the procedure for the investigation of these states. In Sec. III, we study the soliton solutions, their stability, and dynamics in a number of different parameter regimes. The findings are summarized in Sec. IV. In the Appendix, we outline a perturbation-theory analysis that validates and elucidates our numerical findings.

\section{FORMULATION OF THE PROBLEM}

We consider a 2D square-lattice nonlinear-waveguide array consisting of identical regularly spaced elements. Each waveguide carries two circular or linear mutually orthogonal polarization modes that interact nonlinearly via XPM. In a birefringent core, the circular polarizations are linearly coupled [31,32]. A similar situation exists in a twisted core when linear polarizations are involved $[31,33,34]$. In addition to that, one may also consider the case when two light beams have two different wavelengths. In this case, linear coupling is absent and effectively the dynamics of the problem are dictated by nonlinear self-phase modulation (SPM) and XPM interactions.

The appropriately normalized general model, involving all the above-mentioned processes, takes the form

$$
i \frac{d}{d z} \psi_{m, n}=-\Delta_{2} \psi_{m, n}-\left(\left|\psi_{m, n}\right|^{2}+\beta\left|\phi_{m, n}\right|^{2}\right) \psi_{m, n}-\kappa \phi_{m, n}
$$

$$
i \frac{d}{d z} \phi_{m, n}=-\Delta_{2} \phi_{m, n}-\left(\left|\phi_{m, n}\right|^{2}+\beta\left|\psi_{m, n}\right|^{2}\right) \phi_{m, n}-\kappa \psi_{m, n},
$$

$$
\Delta_{2} \psi_{m, n} \equiv C\left(\psi_{m+1, n}+\psi_{m-1, n}+\psi_{m, n+1}+\psi_{m, n-1}-4 \psi_{m, n}\right)
$$

where $z$ is the propagation distance along the waveguides, and $\beta$ is the ratio of the XPM and SPM coefficients. For the interaction between two linear polarizations, $\beta=2 / 3$, whereas for the circular polarizations, $\beta=2$; the latter value applies also to the case where the modes $\psi_{m, n}$ and $\phi_{m, n}$ involve different carrier wavelengths [31]. The linear coupling constant $C$ between adjacent waveguides, which appears in the definition of the 2D discrete Laplacian $\Delta_{2}$ [see Eq. (3)], is related to the effective lattice spacing $h$ as $C$ $\equiv 1 / h^{2}$.

As was mentioned above, $\kappa$ accounts for the linear coupling (if any) among the two modes inside each waveguide. It is obvious that $\kappa$ can be made positive or negative depending on the sign of the birefringence $n_{x}-n_{y}$. When we consider the effect of $\kappa$ on the dynamical properties of solitons we will, for completeness, display the results for both $\kappa>0$ and $\kappa<0$. Note that, for the symmetric soliton, the linear coupling yields negative and positive contributions, respectively, to the system's Hamiltonian in the cases $\kappa>0$ and $\kappa$ $<0$, therefore one may expect that the solitons may be stable in the former case and unstable in the latter case. It will be demonstrated that this is true indeed.
As concerns the application of the general system, Eqs. (1) and (2), to binary BECs trapped in a 2D optical lattice, the evolutionary variable $z$ should be interpreted as time, the XPM coefficient is of the order of unity [21], and the linearcoupling coefficient $\kappa$ is proportional to the intensity of the microwave radiation that induces transitions between the two hyperfine states $[21,22]$.

Before we proceed to identify solitons, it is important to analyze the spectrum of linear modes in the system. Searching for a solution of the linearized equations (1) and (2) in the standard form

$$
\begin{aligned}
& \psi_{m, n}=\psi^{(0)} \exp \left[i\left(\Lambda z+k_{x} m+k_{y} n\right)\right], \\
& \phi_{m, n}=\phi^{(0)} \exp \left[i\left(\Lambda z+k_{x} m+k_{y} n\right)\right]
\end{aligned}
$$

(with $m$ and $n$ are integers) we find that the dispersion relation includes two branches

$$
\Lambda= \pm \kappa-4 C\left[\sin ^{2}\left(k_{x} / 2\right)+\sin ^{2}\left(k_{y} / 2\right)\right] .
$$

As follows from expression (5), there are following two phonon bands in the system's spectrum:

$$
\begin{gathered}
\kappa-8 C<\Lambda<\kappa, \\
-\kappa-8 C<\Lambda<-\kappa .
\end{gathered}
$$

For example, if $\kappa$ is a positive parameter, note that a gap,

$$
-\kappa<\Lambda<\kappa-8 C,
$$

exists between the two bands provided that $\kappa>4 C$.

It is relevant to compare the allowed bands (6) and (7) with a region in the $\Lambda$ space where solitons are, in principle, possible. Exponentially decaying tails of the soliton also obey the linearized version of Eqs. (1) and (2). In the asymptotic region far from the soliton's core, one may naturally expect that the tail becomes asymptotically isotropic (which is corroborated by numerical solutions for solitons, see below) and the linearized equations may be approximated by their continuum-limit form. This implies that the asymptotic shape of the tail, in both $\psi$ and $\phi$ components, is $\psi_{m, n}, \phi_{m, n} \sim\left(m^{2}+n^{2}\right)^{-1 / 2} \exp \left(i \Lambda z-\rho \sqrt{m^{2}+n^{2}}\right)$, where $\Lambda$ is the soliton's propagation constant and $\rho$ is a positive constant. It then follows that the relation between $\Lambda$ and $\rho$ assumes the form

$$
\Lambda= \pm \kappa+C \rho^{2}
$$

Comparison of Eq. (9) with the allowed bands (6) and (7) shows that solitons corresponding to the positive sign of $\kappa$ in Eq. (9) may exist precisely above the upper band, in accordance with the commonly known principle that the propagation constants (or frequencies, in the case of the temporal evolution) of radiation waves and solitons do not overlap, as otherwise solitons will be losing energy through emission of radiation.

Solitons which correspond to the negative sign of $\kappa$ in Eq. (9) may overlap with the upper band, in which case we expect that they do not exist or are unstable [recall that the 
dispersion equation (9) is only a necessary, but not sufficient, condition for the stability of the localized solutions]. Indeed, our numerical solution has never revealed solitons that would overlap with the band (see details below). Note that the solitons corresponding to the negative sign of $\kappa$ in Eq. (9) could potentially exist in the gap of Eq. (8), should the gap be present; however, in this work, we do not consider the case when this gap is possible.

In order to identify vector discrete solitons, we look for stationary solitary-wave solutions of Eqs. (1) and (2), which have the form

$$
\begin{aligned}
& \psi_{m, n}=\exp \left(i \Lambda_{1} z\right) u_{m, n}, \\
& \phi_{m, n}=\exp \left(i \Lambda_{2} z\right) v_{m, n},
\end{aligned}
$$

where $\Lambda_{1}$ and $\Lambda_{2}$ are the propagation constants of the two components of the soliton (they may be different in the absence of linear coupling, see below). The substitution of Eqs. (10) and (11) into Eqs. (1) and (2) yields equations for the real static fields $u_{m, n}$ and $v_{m, n}$,

$$
\begin{aligned}
F\left(u_{m, n}, v_{m, n}\right) \equiv & \Delta_{2} u_{m, n}+\left(\left|u_{m, n}\right|^{2}+\beta\left|v_{m, n}\right|^{2}\right) u_{m, n}+\kappa v_{m, n} \\
& -\Lambda_{1} u_{m, n}=0, \\
G\left(u_{m, n}, v_{m, n}\right) \equiv & \Delta_{2} v_{m, n}+\left(\left|v_{m, n}\right|^{2}+\beta\left|u_{m, n}\right|^{2}\right) v_{m, n}+\kappa u_{m, n} \\
& -\Lambda_{2} v_{m, n}=0 .
\end{aligned}
$$

A numerical solution to Eqs. (12) and (13) (with $\Lambda_{1}$ and $\Lambda_{2}$, generally, different if $\kappa=0$ and with $\Lambda_{1} \equiv \Lambda_{2}$ if $\kappa \neq 0$ ) will be obtained in the following section by means of a Newton iteration method. Here, we only consider vector discrete solitons that involve only in phase $u_{m, n}$ and $v_{m, n}$ components (unstaggered) for stability reasons as these states tend to minimize the system's Hamiltonian.

Once the solution is obtained, we will perform linear stability analysis around it, looking for perturbed solutions as [35-38]

$$
\begin{aligned}
\psi_{m, n}= & \exp \left(i \Lambda_{1} z\right)\left[u_{m, n}+\epsilon a_{m, n} \exp (i \omega z)\right. \\
& \left.+\epsilon b_{m, n} \exp \left(-i \omega^{*} z\right)\right], \\
\phi_{m, n}= & \exp \left(i \Lambda_{2} z\right)\left[v_{m, n}+\epsilon c_{m, n} \exp (i \omega z)\right. \\
& \left.+d_{m, n} \exp \left(-i \omega^{*} z\right)\right],
\end{aligned}
$$

where $\epsilon$ is the infinitesimal amplitude of the perturbation and $\omega$ is the eigenvalue corresponding to the linear (in)stability mode.

Thus, the path that is followed in the following section for the numerical investigation of Eqs. (1) and (2) can be summarized as follows:

(1) We first solve Eqs. (12) and (13) numerically.

(2) Then the linear stability eigenvalue problem is solved, and the eigenvalues and eigenstates are obtained.

(3) If the computation of the eigenvalues indicates the presence of an instability, we perform numerical integration of the Eqs. (1) and (2) with the numerically exact unstable solution taken as the initial configuration (in some cases, a small perturbation proportional to the unstable eigenmode is added, in order to accelerate the development of the instability). The objective is to monitor the evolution of the instability.

These steps will be carried out for four different relevant sets of parameter values, namely: (i) $\beta=0, \kappa \neq 0$, and $\Lambda_{1}$ $=\Lambda_{2} \equiv \Lambda$ ( $\Lambda$ will be kept fixed); (ii) $\beta=2$ or $2 / 3, \kappa=0$, and $\Lambda_{1} \neq \Lambda_{2}$, which corresponds to the array carrying two polarizations of light (linear if $\beta=2 / 3$ or circular if $\beta=2$, in both cases without linear mixing between the polarizations) or two different wavelengths $(\beta=2)$. The same corresponds to the binary-BEC trapped in the optical lattice without the resonant coupling between components. (iii) Arbitrary $\beta$ $\neq 0, \kappa=0$, and $\Lambda_{1}=\Lambda_{2} \equiv \Lambda$ ( $\Lambda$ will be kept fixed), which is a more formal case, added for the completeness of the study of the model. (iv) $\beta=2,2 / 3, \kappa \neq 0$, and $\Lambda_{1}=\Lambda_{2}$ ( $\Lambda$ will be kept fixed), which is the most general case. It includes, if $\beta=2$, two polarizations with the linear mixing between them. Another interpretation is the binary-BEC model with the coupling between the components induced by a resonant radiation field. In the case of BEC, $z$ stands for time. In fact, the above cases, (i)-(iv), represent one-parameter cuts through the parameter space of each physical version of the model at a fixed value of the soliton's propagation constant $s$. It has been found that these cuts display the basic phenomenology of the system in the generic form (we have also performed the analysis at other values of $\Lambda_{1,2}$, concluding that results are very similar to those presented in the paper). In most cases, the cuts are made at fixed values of the propagation constant, but by varying the linear-coupling constant $\kappa$. The purpose of this mode of presentation of results is to demonstrate the role of the strength of the linear coupling while the size of the soliton, which can be roughly estimated as $\Lambda^{-1 / 2}$, remains constant. As mentioned above, the stability analysis, in terms of the eigenvalues of small perturbations, is an important ingredient of the consideration. Therefore, it is relevant to describe here what types of the eigenvalues may be expected.

For a stable discrete soliton in the one-component model (obtained, for instance, by setting $\phi_{m, n}=0$, provided that $\kappa$ $=0$ ), there is a pair of perturbation eigenmodes with zero eigenfrequencies. These modes are generated by the phase (gauge) invariance of the equations, which is, in turn, related through the Noether's theorem to the conservation of the norm of the solution, $\Sigma_{m, n}\left|\psi_{m, n}\right|^{2}$ [39] (in optical waveguide arrays, the norm has the physical meaning of the net power).

For the perturbations about the stationary solution in the one-component model, one might also expect the existence of a pair of translational modes [40]. However, since the translational invariance is broken by the discreteness, the corresponding eigenvalues $\omega_{\text {tr }}$ are different from zero. A bifurcation giving rise to the latter modes in 1D systems has been quantified in Refs. [41-43], and it has been found that the eigenvalues vanish exponentially as the lattice spacing $h$ approaches zero, $\omega_{\mathrm{tr}} \sim \exp \left[-\pi^{2} /(2 h)\right]$ (recall that $h$ $\equiv C^{-1 / 2}$ ). 
Finally, there is a continuous spectrum of eigenvalues, associated with the zero solution $\left(\psi_{m, n}=0\right)$. The continuous spectrum of the one-component model consists of planewave eigenfunctions $\sim \exp \left[i\left(k_{x} n+k_{y} m-\omega z\right)\right]$ and satisfies the dispersion relation (in 2D) $\omega= \pm\left[\Lambda_{1}+2 C\left(2-\cos k_{x}\right.\right.$ $\left.\left.-\cos k_{y}\right)\right]$.

For the two-component model, similar features are present. In particular, if $\kappa=0$, there are four zero eigenmodes; in this case, there are two conserved norms (powers), one for each component. On the contrary, if $\kappa \neq 0$, it is evident that only the sum of the two powers, $\Sigma_{m, n}\left(\left|\psi_{m, n}\right|^{2}\right.$ $\left.+\left|\phi_{m, n}\right|^{2}\right)$, is conserved. Hence, as soon as $\kappa$ becomes different from zero, one of the eigenvalue pairs at the origin has to bifurcate away. Besides that, there are two nonzero translational-eigenvalue pairs $\omega_{\text {tr }}$ (one pertaining to each component of the stationary pulse). Lastly, there are the continuous-spectrum branches, obeying the dispersion relations

$$
\begin{aligned}
& \omega= \pm\left[\Lambda_{1} \pm \kappa+2 C\left(2-\cos k_{x}-\cos k_{y}\right)\right], \\
& \omega= \pm\left[\Lambda_{2}-\kappa+2 C\left(2-\cos k_{x}-\cos k_{y}\right)\right] .
\end{aligned}
$$

On the basis of this information about the spectrum, a perturbative technique can be developed to study the behavior of the eigenvalues of the single-component DNLS model upon the perturbation imposed by the coupling to the second component. Technical details of this approach are presented in the Appendix. The main results obtained by means of this technique are summarized below in Eq. (A17) and, for the specific cases of interest, in Eqs. (A21). These results will be discussed in detail below.

\section{NUMERICAL RESULTS}

\section{A. The system with linear coupling}

We now examine the nature and stability of solutions for the four cases specified in the preceding section. The first case we consider has parameter values $\beta=0, \kappa \neq 0$, and $\Lambda_{1}=\Lambda_{2}$. We fix $h \equiv C^{-1 / 2}=0.75$ and $\Lambda_{1}=\Lambda_{2}=2$, and vary $\kappa$ in the interval $-4 \leqslant \kappa<2$. Note that both positive and negative values of the linear-coupling constant $\kappa$ are physically meaningful (the same pertains to the birefringenceinduced linear mixing between two circular polarizations, see, e.g., Ref. [32]). We stress that no soliton solutions have been found, for fixed $\Lambda_{1}=\Lambda_{2}=2$, in the case $\kappa>2$, in precise agreement with the fact that solitons cannot exist with $\Lambda<\kappa$, see Eq. (9).

Results known from the 1D continuиm model of the dualcore nonlinear optical fiber [44] suggest that solution of two different types may be expected in this case: obvious symmetric ones, with identical field configurations in the two components, and nontrivial solutions with a spontaneously broken symmetry. We start the analysis with the symmetric solitons.

For $\kappa>0$, we find that, as one of the two phase invariances is broken (as discussed in the preceding section), only one of the two phase eigenvalue pairs remains at the origin. The other pair immediately undergoes a bifurcation along the imaginary axis, yielding an instability of the symmetric soliton, which sets in at $\kappa=0$. As $\kappa$ is increased, the amplitude of the solution decreases and its width increases, so that the norm of the solution decreases, as is shown in panel (a) of Fig. 1. At $\kappa \approx 0.24$ (corresponding to the minimum in the inset of Fig. 1 and implying a saddle-node bifurcation), an additional pair of eigenfrequencies, that has bifurcated from the continuous spectrum, also becomes imaginary, adding to the instability of the configuration [see panel (b) of Fig. 1 for $\kappa=0.3]$. At this point, we see that the norm of the solution begins to increase with $\kappa$. Finally, as $\kappa$ is further increased, the first imaginary pair starts moving towards the real axis (at $\kappa \approx 1.0$ ). During its return to the real axis, the pair collides with the second pair of imaginary eigenvalues. The two pairs then continue to approach the real axis together and become real at $\kappa=1.7$. Therefore, at $\kappa>1.7$, the symmetric soliton is stable, in analogy with what is known about the continuum dual-core model [44], see a detailed comparison given below.

We simulated the dynamical evolution of the symmetric soliton in the case in which it is unstable. We have found, as shown in the last two panels of Fig. 1, that the unstable solution evolves into a state oscillating around an asymmetric configuration, in which most of the power is contained in one of the two components. The corresponding stationary asymmetric configuration (whose existence is expected, as it was mentioned above) has been numerically identified and found to be stable at all the values of the parameters at which it was investigated $(0<\kappa \leqslant 0.4)$. In particular, for $\kappa=0.3$ (the same value as that used in Fig. 1), the asymmetric configuration and its linear-stability eigenvalues are shown in Fig. 2.

In the case $\kappa<0$, contrary to what was the case for $\kappa$ $>0$, the symmetric beam steepens as $|\kappa|$ is increased and the norm of the solution increases; see panel (a) of Fig. 3. The eigenvalue bifurcation in this case is along the real axis, hence no instability sets in for very small $|\kappa|$. However, very "soon"—at $\kappa=-0.0025$ - the eigenvalues collide with those corresponding to the translational modes (which, at these values of the parameters, were located in the gap between the origin and the continuous spectrum), moving towards the origin. This event leads to an oscillatory [45-48] (alias Hamiltonian Hopf [49]) bifurcation to instability through the generation of a quartet of genuinely complex eigenvalues; recall that, due to the Hamiltonian nature of the problem, when $\omega$ is an eigenfrequency, so are $-\omega, \pm \omega^{\star}$, which together constitute the quartet. This behavior can be observed in the spectral plane $\left(\omega_{r}, \omega_{i}\right)$ of panel (b) of Fig. 3 for $\kappa=-0.45$. Eventually, the eigenvalues return to the continuous band at $\kappa=-3.4$ after a tortuous path, but as discussed in Ref. [46], this is a finite-size effect. In the case of the infinite domain, the eigenvalues return to the axis only for more negative values of $\kappa$. In the latter case, the return occurs beyond the upper-band edge of the continuous spectrum (as opposed to the return that occurs inside the gaps of the band in the finite system).

Simulating the dynamical evolution of the instability (see the bottom panels in Fig. 3), we typically find that, after some oscillations (associated with the nature of the instability), the 

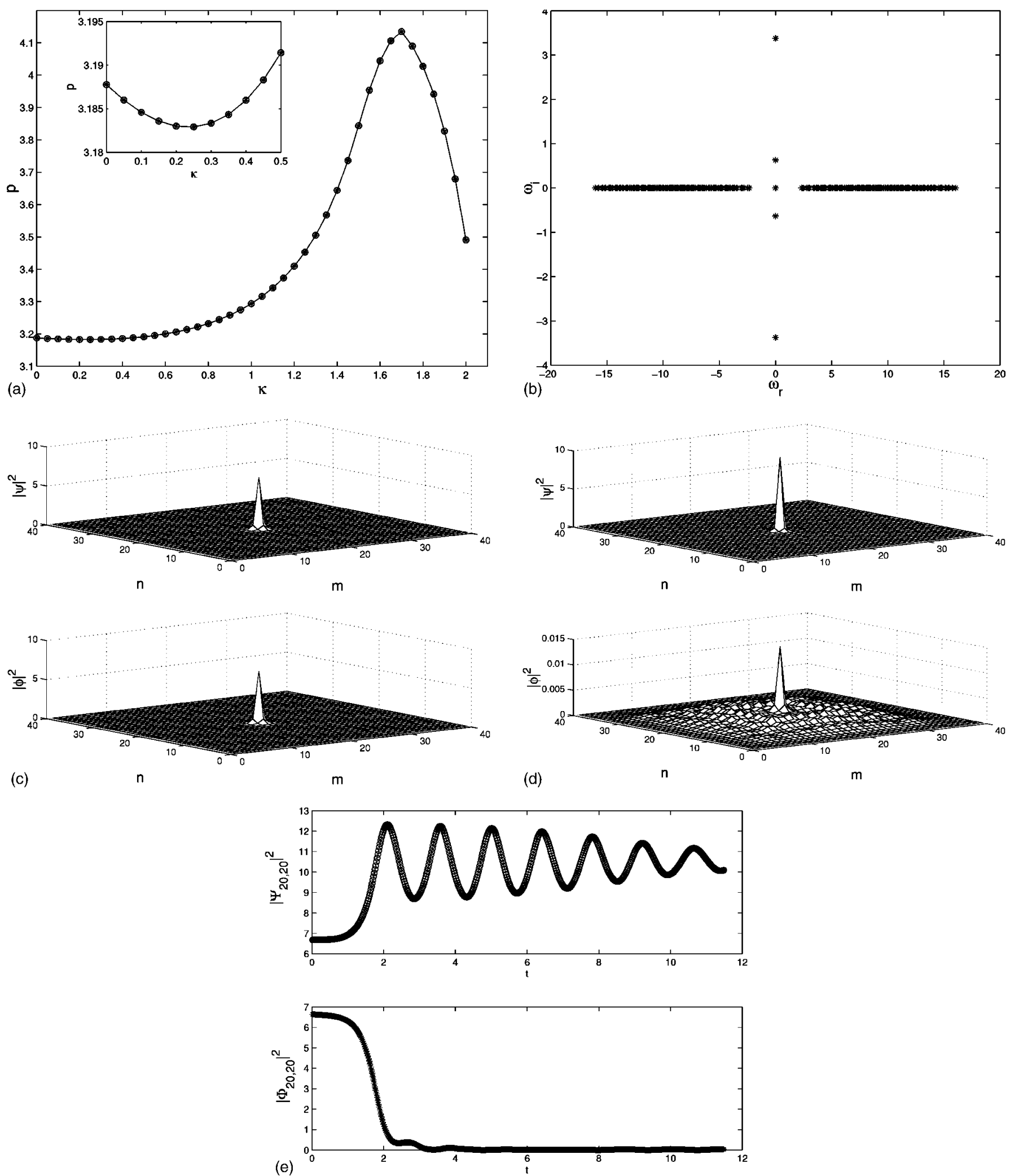

FIG. 1. Panel (a) shows how the norm $(p)$ of each component of the symmetric soliton solution changes with $\kappa$. The inset shows that the norm decreases for small values of $\kappa$, but increases for $\kappa \geqslant 0.24$. The second panel shows the $\left(\omega_{r}, \omega_{i}\right)$ spectral plane of the stability eigenvalues for the same solution for $\kappa=0.3$ (the subscripts refer to the real and imaginary parts of the "eigenfrequency"). An eigenfrequency with a nonzero imaginary part indicates [as per Eqs. (14) and (15)] the presence of an instability. Panels (c) and (d) show, respectively, the two fields at the values of the propagation distance $z=0$ and $z=6$, for $\kappa=0.3$. The bottom panel shows the squared absolute values of the field in the two components at the central site of the pulse $[(m, n)=(20,20)]$ as functions of $z$. The latter picture suggests that the solution is attempting to transform itself into a stable asymmetric solution. 

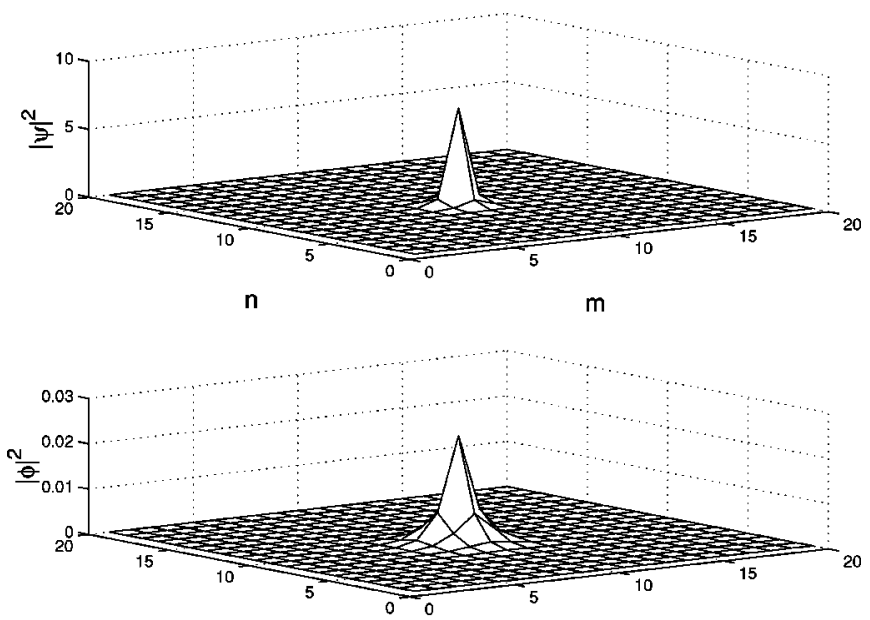

(a)

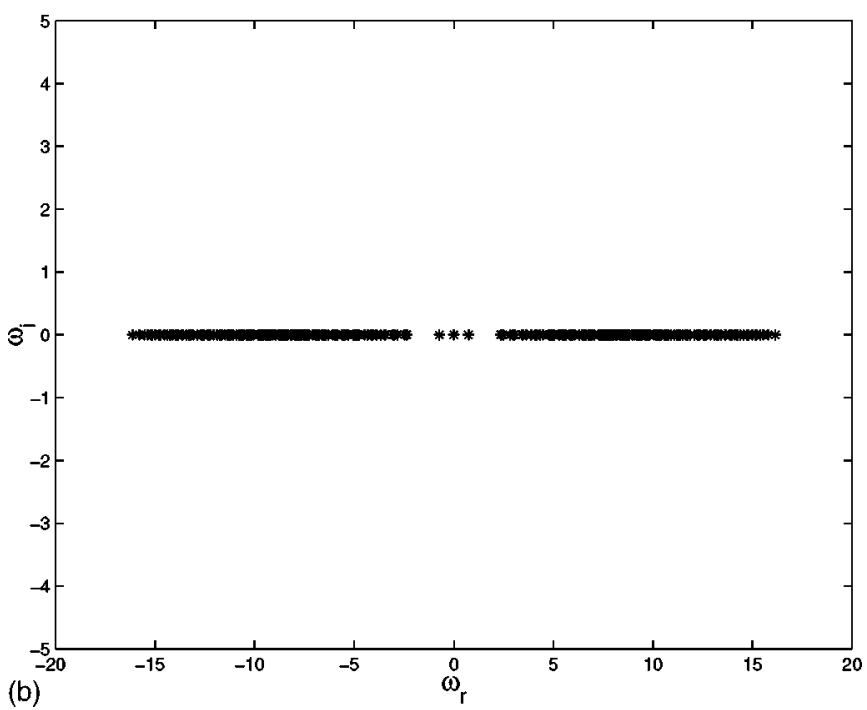

FIG. 2. The asymmetric solution and its linear stability picture are shown for $\kappa=0.3$ (same as for Fig. 1). One can observe that, contrary to what is the case for the symmetric configuration at the same values of the parameters, the asymmetric one is stable, which explains the above-mentioned observation, that the development of the instability of the symmetric solution leads to oscillations around a stable asymmetric configuration.

soliton of the present type (corresponding to $\kappa<0$ ) does not rearrange itself into a stable one. Instead, it completely decays into small-amplitude radiation waves.

The general results for the stability and instability of the symmetric and asymmetric solitons presented above are quite similar to those known in the above-mentioned 1D continuum model of the dual-core nonlinear optical fiber [44]. Indeed, for a fixed power (norm) of the solution, the symmetric soliton is unstable at small values of the linearcoupling constant, and becomes stable via a pitchfork bifurcation if the coupling constant exceeds a certain threshold value. Accordingly, in the case when the symmetric soliton is unstable, there exist two stable asymmetric solitons, which are mirror images of each other. Lastly, the symmetric soliton with negative $\kappa$, which is tantamount to an antisymmetric $(\phi=-\psi)$ soliton with $\kappa>0$, is known to be practically always unstable. In the lattice model, coexistence between stable symmetric and asymmetric solitons has not been found, i.e., the bifurcation transforming the symmetric soliton into asymmetric ones, appears to be supercritical in the latter model.

Naturally, the question arises as to whether some of the features observed in the case of $\kappa \neq 0$ (and especially for small $\kappa$ ) can be explained analytically. One thing that can be shown [50] for $\kappa \neq 0$ is that, if a real (stable) eigenvalue pair bifurcates (which was reported above for the case $\kappa<0$ ), the Krein signature of this eigenvalue [defined as $K=$ $-\operatorname{sgn}\left(\Sigma_{k} U_{k} W_{k}\right)-$ see Refs. [36,45,51,52], and for the definition of the eigenvector components $U_{k}$ and $W_{k}$, see the Appendix] takes the form

$$
K=\operatorname{sgn}\left(\kappa \sum_{k} W_{k}^{2}\right) .
$$

Hence, for $\kappa<0$, the bifurcating eigenvalue has a negative Krein sign, which, according to Refs. [51,52], indicates that the configuration is structurally unstable, and upon a collision of this eigenvalue with other isolated or continuousspectrum eigenvalues, an oscillatory instability will set in [45,51]. We have also checked the validity of Eq. (A20) (see the Appendix) in this case. We have found (by performing the relevant summations in a number of numerical experiments) that for the cases considered $\Sigma_{k} U_{k}^{2} u_{k}^{2}>\Sigma U_{k} u_{k}$ and hence, for negative $\kappa$ (and small $\kappa$ ), the linear terms are dominant and give a positive contribution [hence, the eigenfrequency must be real, as it is observed], while for positive $\kappa$, they will lead to a bifurcation along the imaginary axis [the right-hand side of Eq. (A20) will be negative], as is observed indeed in the numerical experiment. In either case, for large values of $\kappa$, the $\kappa^{2}$ term takes over and in the case of $\kappa>0$, the imaginary eigenvalue pair due to the broken phase symmetry moves towards the origin, while in the $\kappa$ $<0$ case, the eigenvalue moves further away from the origin. Notice, however, that the leading-order perturbative result gives correct qualitative behavior, even though it cannot, $a$ priori, be regarded as being valid for large $\kappa$.

\section{B. Systems with nonlinear SPM and XPM interactions}

We now consider a case with zero linear-coupling and nonzero XPM coefficients: $\beta=2, \kappa=0$, and $\Lambda_{1}>\Lambda_{2}$ (recall this case pertains to two circular polarizations or two carrier wavelengths in optics, as well as to binary BECs trapped in an optical lattice, without resonant radiation field inducing transitions between the two components). We set, in particular, $h \equiv C^{-1 / 2}=0.75$ and $\Lambda_{1}=3$, and gradually decrease $\Lambda_{2}$ from the initial value $\Lambda_{2}=3$. We observe that, in this case, the second component grows in amplitude (as well as in norm), while the first component gradually decreases its amplitude and norm, as is shown in panel (a) of Fig. 4. In this case, since $\kappa=0$, both phase eigenvalue pairs are at the origin and both norms are conserved. This implies that no oscillatory instability can arise in this setting. As is well known from numerical experiments in $1 \mathrm{D}$ and 2D [36-38] (see also Ref. [50]), the translational modes have the same 

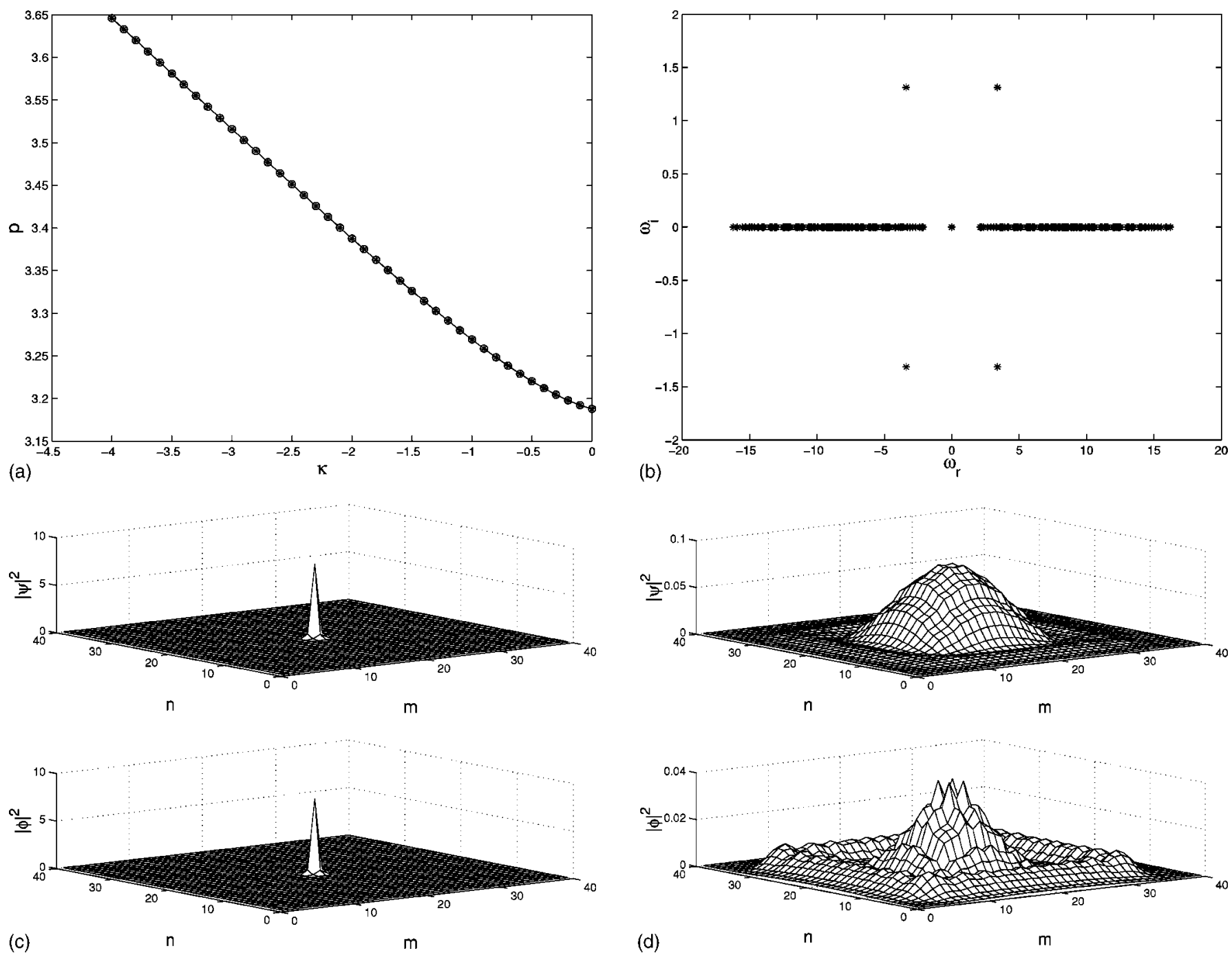

(d)

FIG. 3. Panel (a) shows that the norm of each component of the symmetric soliton grows, in the case $\kappa<0$, as $|\kappa|$ increases. Panel (b) shows the quartet of the unstable eigenvalues of a soliton of this type in the $\left(\omega_{r}, \omega_{i}\right)$ spectral plane for $\kappa=-0.45$. The left and right parts of the bottom panel show, respectively, the two fields at $z=0$ and $z=8$ for $\kappa=-0.45$, illustrating the destruction of the soliton.

Krein signature as the continuous spectrum, hence they do not result (for the focusing nonlinearity) in oscillatory instabilities. Therefore, the only instability that can occur is via an excursion of the eigenvalue pair of the continuous spectrum through the origin to the imaginary axis. This was, in fact, observed to occur at $\Lambda_{2}=1.35$; see, e.g., panel (b) of Fig. 4 for $\Lambda_{2}=0.6$. In this case, the simulated dynamical evolution of the instability leads to the total decay of the soliton into lattice "phonons," but without the oscillatory transient regime, which was found in the case of the instability in the preceding paragraph.

In the case of $\Lambda_{2}>\Lambda_{1}\left(\Lambda_{1}\right.$ is once again fixed to be 3$)$, the first component of the soliton is the one that grows with $\Lambda_{2}$, whereas the second component decreases its amplitude and norm, as shown in panel (a) of Fig. 5. In this case, the eigenfrequencies move outwards (as opposed to the inward motion reported above for the case $\Lambda_{2}<\Lambda_{1}$ ), hence no instability is observed for increasing values of $\Lambda_{2}$.

The evolution of the continuous spectrum eigenvalues in this case can be directly predicted from Eqs. (16) and (17).
Since the two bands of the continuous spectrum consist of the intervals $\omega \in \pm\left[\Lambda_{1}, \Lambda_{1}+8 C\right]$ and $\omega \in \pm\left[\Lambda_{2}, \Lambda_{2}\right.$ $+8 C]$, the motion of the band edges can be seen to be in agreement with the numerical findings presented above. In fact, we have also checked that, for $\Lambda_{2}>\Lambda_{1}+8 C$, the two bands separate and the continuous spectrum consists of two distinct intervals.

The situation is different in the case where the XPM coefficient takes the other physically relevant value, $\beta=2 / 3$ instead of $\beta=2$ (and again $\kappa=0$; recall $\beta=2 / 3$ corresponds to the linear polarizations). In this case, for $\Lambda_{1}>\Lambda_{2}$, it is the first (rather than the second as in the case $\beta=2$ ) component of the soliton that grows in its amplitude and norm. Vice versa, for $\Lambda_{1}<\Lambda_{2}$, it is the second (rather than the first as in the case $\beta=2$ ) component that grows. In fact, these two cases $(\beta=2 / 3$ and $\beta=2)$ clearly demonstrate a contrast between the cases of the weak $(\beta=2 / 3)$ and strong $(\beta=2)$ XPM couplings between the components. Similarly to what is obvious in the absence of the coupling, in the weakcoupling case, the decrease of the frequency of one compo- 

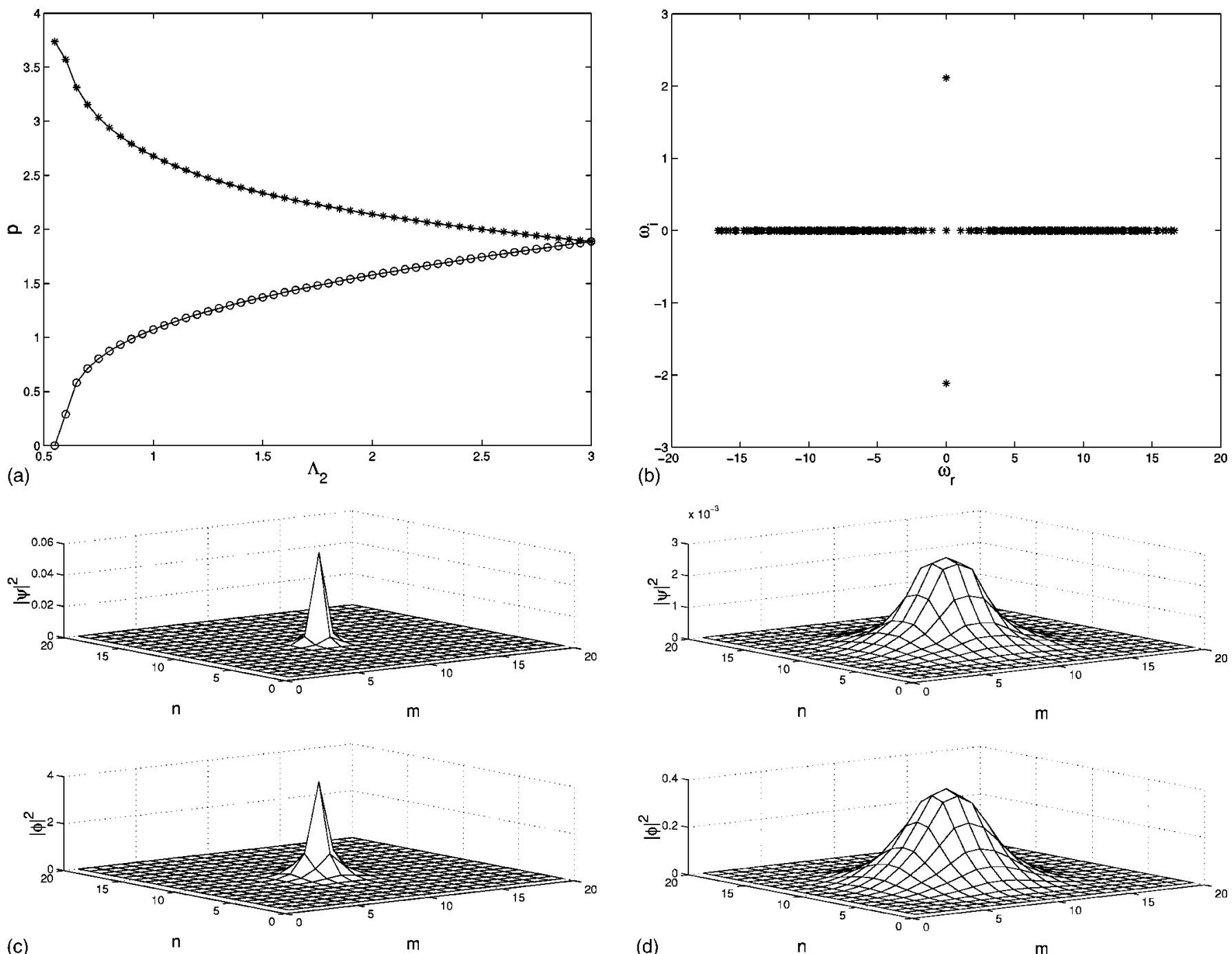

(c)

m

(d)

FIG. 4. Panel (a) shows that the norm of the first component of the soliton (the line with circles) decreases, whereas the norm of the second component (the line with asterisks) increases by the same amount as $\Lambda_{2}$ decreases. The second panel shows the $\left(\omega_{r}, \omega_{i}\right)$ spectral plane of eigenfrequencies for $\Lambda_{2}=0.6$. The bottom panels show the result of the simulations of the instability development for an unstable soliton: the left and right parts show the field configurations at $z=0$ and $z=4.0$, respectively, for $\Lambda_{2}=0.6$.

nent reduces the amplitude and the norm of that same component. However, for the strong coupling the situation is reversed, the decrease of the frequency of a given component causing the decrease of the amplitude and norm of the other component. Thus, there must exist a critical value $\beta_{\mathrm{cr}} \simeq 1$, beyond which the "reaction" of the soliton solutions to the variation of the frequencies is reversed.

A dynamical feature that is common to both cases, $\beta$ $=2 / 3$ and $\beta=2$, is that the continuous spectrum eigenvalues move inwards for $\Lambda_{2}<\Lambda_{1}$ and outwards in the opposite case. However, the weakness of the coupling in the case $\beta=2 / 3$ does not allow instability for $\Lambda_{2}<\Lambda_{1}$. In fact, around $\Lambda_{2}$ $\approx 0.8$, the second component of the soliton almost disappears, and the numerical computations show that the eigenvalues corresponding to the phase invariance of the wave in the second component bifurcate from the origin and move towards the corresponding band of the continuous spectrum. For $\Lambda_{1}<\Lambda_{2}$, the same feature is observed as the first com- ponent of the soliton becomes almost flat (very broad) for $\Lambda_{2} \approx 6.7$.

Comparing the above results with what is well known for the continuum 1D model of the bimodal nonlinear optical fiber, we note that, in the latter model, all the vectorial solitons are stable [53]. Thus, the possible instability is a specific feature of the lattice model.

We now move on to the case with arbitrary $\beta \neq 0$, while $\kappa=0$ and $\Lambda_{1}=\Lambda_{2}$. Arbitrary values of $\beta$ are not of direct physical relevance, but we study this case here for reasons of completeness of the exposition.

For $\beta<0$, we start with $\Lambda_{1}=\Lambda_{2}=5$ and $h \equiv C^{-1 / 2}$ $=0.55$; this relatively small $h$ is used to probe the translational modes. In particular, for $h=O(1)$, the translational eigenfrequencies have already merged with the continuous spectrum; due to their Krein signature being the same as that of the continuous band, they do not create instabilities, but just immerse into the continuous spectrum of eigenvalues. 

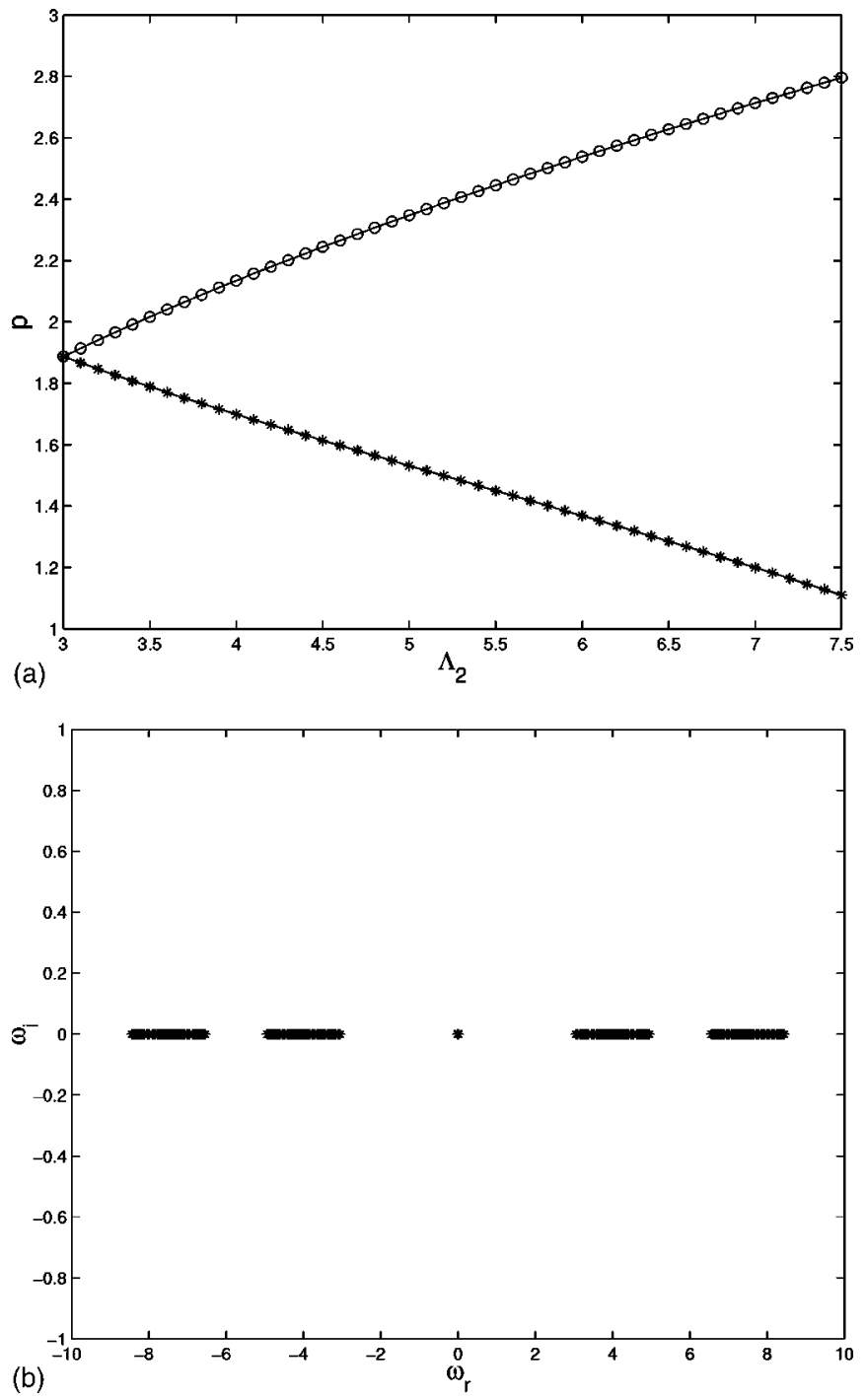

FIG. 5. The top panel shows that the norm of the first component (the line with circles) increases, whereas the norm of the second component (the line with asterisks) decreases by the same amount as $\Lambda_{2}$ increases. The bottom panel shows two separated bands of the continuous spectrum. In this case, the spacing is chosen to be $h=2(C=0.25)$, in order to see the band separation for reasonably small values of $\Lambda_{1}$.

However, for smaller $h$ such as used here, they are still in the gap and their behavior under the perturbations can be observed. In particular, for $\beta<0$, one of the two pairs of the translational eigenvalues moves towards the origin; as a result, the eigenfrequencies rapidly find themselves on the imaginary axis. The critical $\beta$ for which they become unstable is $\approx-0.1987$. Notice that in this case, the amplitude of the solution (and its norm) increases as $\beta$ becomes more negative, as it is shown in panel (a) of Fig. 6. Simulations of the dynamical evolution of this instability lead, essentially, to the breaking of the soliton's symmetry, which eventually destroys one of its components and leaves, as an asymptotic state, a nonlinear solitary wave plus lattice radiation in one component and solely the radiation in the other one, as is shown in the lower part of Fig. 6.
On the contrary, for $\beta>0$, once again one of the translational eigenfrequency pairs moves, but this time it moves towards the continuous-spectrum's band edge and no instability arises in the analysis. Parallel to this, the amplitude and norm of the two components decrease.

We can now compare these numerical findings to the predictions of Eq. (A22) (see the Appendix). In particular, the first term of the equation is the unperturbed frequency of the translational mode. Due to the positive Krein sign (i.e., $-\Sigma_{k} U_{k} W_{k}>0$ ) of the translational mode, the second quantity in the expression is positive, while the third one is negative definite. Numerical computations of the two components show that, for the cases considered, $-\omega_{t} \Sigma U_{k} W_{k} u_{k}^{2}$ $>2 \Sigma U_{k}^{2} u_{k}^{2}$, hence, the eigenvalue moves to the right for $\beta$ $>0$ and to the left for $\beta<0$. These predictions are in agreement with the numerical findings.

\section{Systems with both linear coupling and nonlinear interactions}

Finally, we examine the case where both $\beta$ and $\kappa$ are nonzero; as it was explained above, this case, with $\beta=2$, corresponds to a binary BEC trapped in the optical lattice and the linear coupling being induced by the resonant radiation field. For $\beta=2$ and $\kappa>0\left(\Lambda_{1}=\Lambda_{2}=4\right.$ and $h \equiv C^{-1 / 2}$ $=0.75$ ), we find that the amplitudes (and norms) of the beams decrease as $\kappa$ increases. In this case, the phase eigenmodes bifurcate along the real axis due to the large value of the XPM coefficient $\beta$. Notice that this is opposite to the $\beta=0$ case. This is also indicated by Eq. (A25) (see the Appendix), where the addition of the last term of a definite sign for a strong coupling leads the eigenvalue to become real. However, the Krein signature of the eigenvalue is still determined by $\kappa$, being positive in this case, hence no oscillatory instabilities are expected to arise. In fact, eigenvalues bifurcate from the continuous spectrum, collide with the eigenvalues of the phase modes (passing through them), and eventually reach the origin, exiting as an unstable pair at $\kappa$ $\approx 2.25$. After an excursion along the imaginary axis, this unstable pair returns to the real axis and for large $\kappa(\kappa$ $\geqslant 3.7$ ), the soliton is stable again.

This rather unusual situation is reminiscent of what happens in the case of gap solitons in nonlinear Bragg fibers $[54,55]$. Indeed, both an approximate stability analysis, based on the variational approach [56], and direct numerical computations [57] demonstrate that the gap is split into regions where the solitons are stable and unstable. In any case, the stability results obtained here for the 2D discrete solitons suggest to search for these solitons experimentally in the array as they are expected to be stable in a large parametric region, and the power necessary for the existence of the solitons can be lowered by employing a stronger linear coupling (larger $\kappa$ ).

On the contrary, for $\beta=2$ and $\kappa<0$, once again the last term in Eq. (A25) dominates and the phase eigenvalues are imaginary (unstable). When $\kappa \approx-2.5$, these eigenvalues begin to return to the real axis. However, at $\kappa \approx-3.6$, a second pair of eigenvalues, which has bifurcated from the continu- 

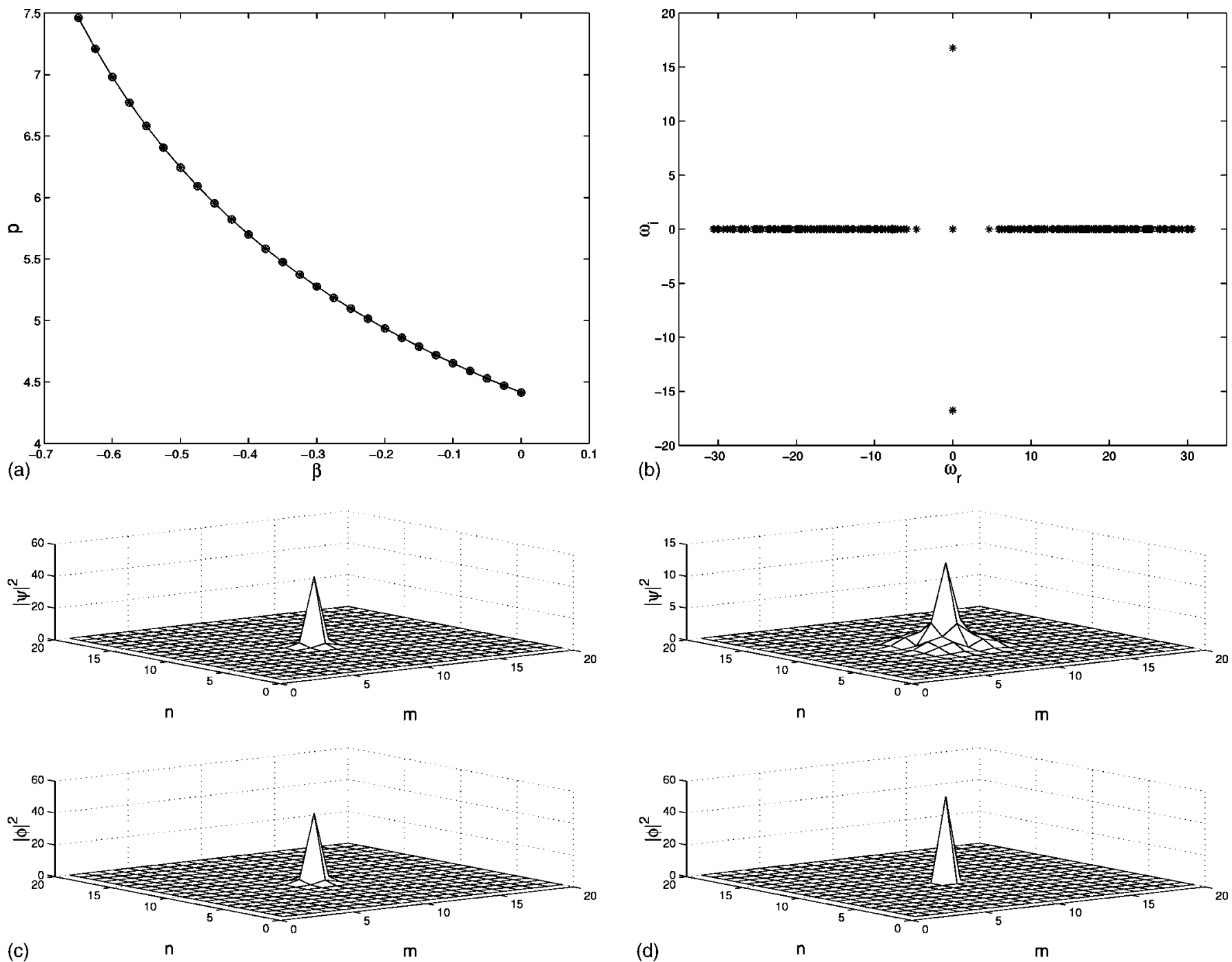

FIG. 6. Panel (a) shows how the norm of the solution increases as $\beta$ becomes more negative. The second panel shows the presence of imaginary eigenfrequencies in the $\left(\omega_{r}, \omega_{i}\right)$ spectral plane at $\beta=-0.65$. The bottom panels show the two components of the simulated solution at $z=0$ and $z=0.6$ in the left and right parts for $\beta=-0.65$.

ous spectrum, becomes imaginary [see panel (a) of Fig. 7]. The two pairs collide on the imaginary axis and form a complex quartet at $\kappa \approx-3.8$ [see panel (c) of Fig. 7]. In this case, the norm and amplitude of both components increase as is shown in panel (a) of Fig. 7. As for the continuous spectrum, it moves towards the origin as $\kappa$ becomes more negative. Finally, simulations of the evolution of the unstable soliton in the model with $\beta=2$ and $\kappa \neq 0$ always show its complete destruction, as is displayed in panels (d) and (e) of Fig. 7.

For the weak XPM case, $\beta=2 / 3$ (this case is less interesting physically, but it is meaningful, corresponding to the set of two linear polarizations with linear mixing between them due to the fiber twist), the last term in Eq. (A25) is not as significant. For $\kappa>0$, even though once again the beam decreases in amplitude (and widens), the eigenvalues move along the imaginary axis. They eventually return from this excursion and become real once again at $\kappa \geqslant 1.35$. The modes at the edge of the continuous spectrum, however, also bifurcate, eventually becoming imaginary for $\kappa>2.3$. This pair of eigenfrequencies also returns to the real axis when $\kappa \approx 3.8$. The latter instability is dynamically manifested (in the simulations) through fast destruction of the soliton.

In the case of $\kappa<0$ and $\beta=2 / 3$, it happens again that the first set of terms in Eq. (A25) dominates over the last term, and the eigenfrequencies become real. But as in the case $\beta$ $=0$, this occurs with the "wrong" (i.e., negative) Krein signature, and the eventual collision of the eigenvalues with the continuous band yields an oscillatory instability. This is shown in the spectral-plane picture in panel (b) of Fig. 8. Panel (a) shows the increase in the amplitude and norm of the pulse (as $\kappa$ becomes more negative), panels (c) and (d) show, after some transient oscillatory behavior, the manifestation of the instability for $\kappa=-1.0$ through the destruction of both components of the soliton.

\section{CONCLUSIONS AND FUTURE CHALLENGES}

We have explored the existence and the stability of twodimensional discrete vector solitons in several models of 

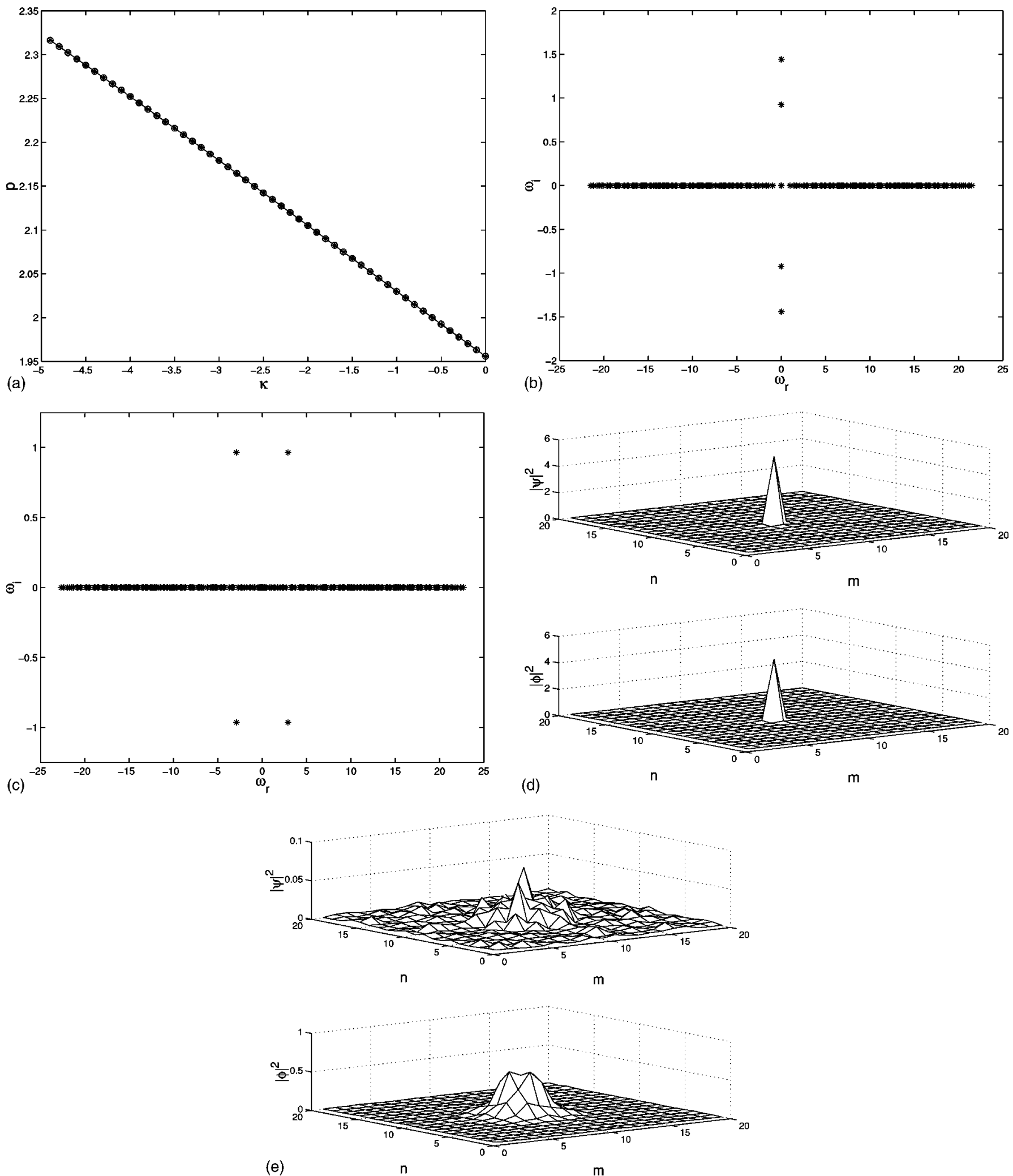

(e)

m

FIG. 7. Panel (a) shows that the norm of the components increases as $\kappa$ becomes more negative. Panels $(\mathrm{b})$ and $(\mathrm{c})$ show the $\left(\omega_{r}, \omega_{i}\right)$ spectral plane for $\kappa=-3.7$ and $\kappa=-4.9$, respectively. Panels (d) and (e) show the two components of the simulated unstable solution at $z=0$ and $z=60.5$, respectively, for $\kappa=-4.9$. 

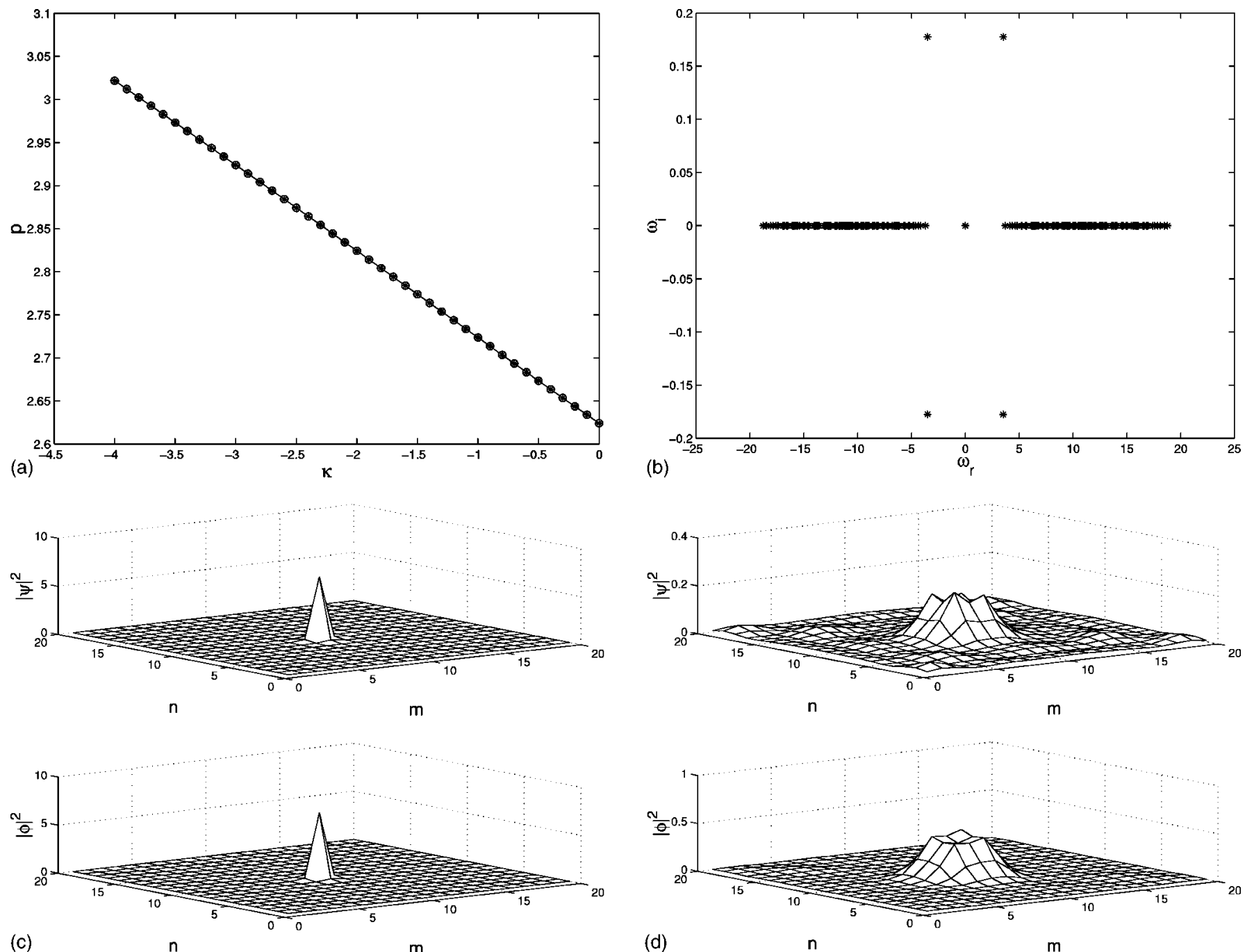

(d)

n

m

FIG. 8. Panel (a) shows that the norm of the components increases as $\kappa$ becomes more negative. Panel (b) shows the $\left(\omega_{r}, \omega_{i}\right)$ spectral plane when $\kappa=-1.0$. The left and right parts of the bottom panel show the two components of the simulated unstable solution at $z=0$ and $z=35.3$, respectively, for $\kappa=-1.0$.

nonlinear optical waveguide arrays. The vector interactions in these systems are described by two coupled discrete nonlinear Schrödinger equations. We considered the impact of $\mathrm{XPM}$, as well as of linear coupling, on the two vector components. The same general model applies to two-component Bose-Einstein condensates trapped in a 2D optical lattice (in particular, the linear coupling between the two equations accounts for Rabi or Josephson oscillations between two hyperfine states of BEC atoms induced by microwave radiation). Instabilities and parameter regions in which they occur were found. The corresponding regimes of weak and strong couplings were identified, and differences in the relevant phenomenology were highlighted. Numerical simulations were performed for unstable states in order to investigate their dynamical evolution. It was observed, depending on the parameters, that unstable solitons either transform themselves into stable asymmetric ones or are completely destroyed.

Naturally, many questions still remain unaddressed for such models. In particular, in this work, we have only concerned ourselves with single-humped solutions, the socalled $|0,0\rangle$ solutions in the notation of Ref. [58] (the terms in the bracket denote the number of nodes in the field describing each component). It would be interesting to extend the consideration to multihumped solutions and, in particular, to $|0,1\rangle$ and/or $|1,1\rangle$ solutions, and dipole solutions [29], which have recently been observed experimentally in continuum media with saturable nonlinearities, see, e.g., Ref. [30].

Note also that, in the discrete setup, vortexlike solutions are not necessarily unstable, as they are in the continuum limit. In fact, for sufficiently weak coupling between the lattice sites, discrete vortices have been found and shown to be stable in one-component DNLS-type equations in Refs. $[59,60]$. Identifying the existence, stability and dynamics of the vortex, dipole, and more general "excited-state" solutions in the context of 2D nonlinear waveguide arrays seems a natural subject for future investigation. Such studies are particularly interesting, given the robustness of some of these solutions in the recently studied continuum cases [29]. The 
investigation of such solitary waves is currently in progress and will be reported elsewhere.

\section{ACKNOWLEDGMENTS}

We appreciate a valuable discussion with M. Segev. This research is supported by the U.S. Department of Energy, under Contract No. W-7405-ENG-36. J.H. and B.A.M. acknowledge the hospitality of the Theoretical Division and Center for Nonlinear Studies of the Los Alamos National Laboratory. P.G.K. gratefully acknowledges partial financial support from the University of Massachusetts through a Faculty Research Grant and from the Clay Mathematics Institute.

\section{APPENDIX: THE LINEAR STABILITY PROBLEM}

The linear-stability eigenvalue problem is based on the equation

$$
\omega\left(\begin{array}{c}
a_{k} \\
b_{k}^{*} \\
c_{k} \\
d_{k}^{*}
\end{array}\right)=\mathbf{J} \cdot\left(\begin{array}{c}
a_{k} \\
b_{k}^{*} \\
c_{k} \\
d_{k}^{*}
\end{array}\right)
$$

where $\mathbf{J}$ is the linear stability matrix (Jacobian) of the form

$$
\mathbf{J}=\left(\begin{array}{llll}
J^{11} & J^{12} & J^{13} & J^{14} \\
J^{21} & J^{22} & J^{23} & J^{24} \\
J^{31} & J^{32} & J^{33} & J^{34} \\
J^{41} & J^{42} & J^{43} & J^{44}
\end{array}\right)
$$

Elements of the Jacobian are

$$
\begin{gathered}
J_{k, l}^{11}=\left(2\left|u_{k}\right|^{2}+\beta\left|v_{k}\right|^{2}-2 C-\Lambda_{1}\right) \delta_{k, l}+C \delta_{k, l+1}+C \delta_{k, l-1} \\
+C \delta_{k, l+R}+C \delta_{k, l-R}, \\
J_{k, l}^{12}=u_{k}^{2} \delta_{k, l}, \quad J_{k, l}^{13}=\left(\beta u_{k} v_{k}^{*}+\kappa\right) \delta_{k, l}, \quad J_{k, l}^{14}=\beta u_{k} v_{k} \delta_{k, l}, \\
J_{k, l}^{21}=-\left(J_{k, l}^{12}\right)^{*}, \quad J_{k, l}^{22}=-J_{k, l}^{11}, \quad J_{k, l}^{23}=-\left(J_{k, l}^{14}\right)^{*}, \\
J_{k, l}^{24}=-\left(J_{k, l}^{13}\right)^{*}, \\
J_{k, l}^{33}=\left(2\left|v_{k}\right|^{2}+\beta\left|u_{k}\right|^{2}-2 C-\Lambda_{2}^{13}\right) \delta_{k, l}+C \delta_{k, l+1}+C \delta_{k, l}-1 \\
+C \delta_{k, l+R}+C \delta_{k, l-R}^{32}=J_{k, l}^{14}, \quad J_{k, l}^{34}=v_{k}^{2} \delta_{k, l}, \\
J_{k, l}^{41}=-\left(J_{k, l}^{14}\right)^{*}, \quad J_{k, l}^{42}=-\left(J_{k, l}^{13}\right)^{*}, \quad J_{k, l}^{43}=-\left(J_{k, l}^{34}\right)^{*}, \\
J_{k, l}^{44}=-J_{k, l}^{33} .
\end{gathered}
$$

In Eqs. (A3)-(A8), $\delta_{k, l}$ is the Kronecker's symbol and the asterisk stands for complex conjugation. Note also that only one index has been used in these expressions for the 2D fields $u_{m, n}$ and $v_{m, n}$, because we have cast the fields in a vector of length $R^{2}$, where $R \times R$ being the dimension of the lattice used for the numerical computations in the present $2 \mathrm{D}$ problem. The recasting has been performed in a "row by row" fashion, i.e., $u_{1,1} \rightarrow u_{1}, u_{1, R} \rightarrow u_{R}, u_{2,1} \rightarrow u_{R+1}, u_{2, R}$ $\rightarrow u_{2 R}$, and so on [see also, Eq. (12) of Ref. [38]]. The same reshaping is implied in Eq. (A1) for $a_{k}, b_{k}^{*}, c_{k}$, and $d_{k}^{*}$.

Having discussed in Sec. II the basic features of the spectrum, let us now develop the perturbative technique that will allow us to monitor the behavior of eigenvalues of the single-component DNLS equations, once the perturbations stemming from the second component come into effect. It should be noted that the theoretical framework will be developed in a quite general fashion. However, when applying it to the problem at hand, we will restrict ourselves mainly to considerations regarding discrete eigenvalues (namely, the translational and phase eigenvalues). The reason for this restriction is that the continuous-spectrum eigenvalues, as follows from Eqs. (16) and (17), are separated from the imaginary axis (i.e., from unstable eigenmodes) by a distance $\Lambda_{1}$ and $\Lambda_{2}$, respectively, for the two branches. Since, for our purposes, $\Lambda_{1,2}=O(1)$ in most cases, an instability through a bifurcation from the continuous spectrum would require a bifurcation of the strength $O(1)$, which is beyond the realm of the leading-order perturbation-theory considerations elaborated here.

We develop our formalism starting from the linear stability equation. In particular, using in Eq. (A1)

$$
\begin{gathered}
a_{k}=U_{k}-W_{k}, \\
b_{k}=\left(U_{k}+W_{k}\right)^{*}, \\
c_{k}=X_{k}-Y_{k}, \\
d_{k}=\left(X_{k}+Y_{k}\right)^{*},
\end{gathered}
$$

and after algebraic manipulations, we obtain the eigenvalue problem for the set $\left[U_{k}, W_{k}, X_{k}, Y_{k}\right]^{T}$ in the form

$$
\omega\left(\begin{array}{c}
U_{k} \\
W_{k} \\
X_{k} \\
Y_{k}
\end{array}\right)=\mathbf{L} \cdot\left(\begin{array}{c}
U_{k} \\
W_{k} \\
X_{k} \\
Y_{k}
\end{array}\right)
$$

where

$$
\mathbf{L}=\left(\begin{array}{cccc}
0 & L_{1,-} & 0 & L_{12,-} \\
L_{1,+} & 0 & L_{12,+} & 0 \\
0 & L_{21,-} & 0 & L_{2,-} \\
L_{21,+} & 0 & L_{2,+} & 0
\end{array}\right)
$$

In this reduction, we consider, for simplicity, real solutions (i.e., $u_{k}=u_{k}^{*}$ and $v_{k}=v_{k}^{*}$ ). In that case,

$$
\begin{aligned}
L_{1,-}= & -\left(u_{k}^{2}+\beta v_{k}^{2}-2 C-\Lambda_{1}\right) \delta_{k, l}-C \delta_{k, l+1}-C \delta_{k, l-1} \\
& -C \delta_{k, l+R}-C \delta_{k, l-R},
\end{aligned}
$$




$$
\begin{gathered}
L_{1,+}=-\left(3 u_{k}^{2}+\beta v_{k}^{2}-2 C-\Lambda_{1}\right) \delta_{k, l}-C \delta_{k, l+1}-C \delta_{k, l-1} \\
-C \delta_{k, l+R}-C \delta_{k, l-R}, \\
L_{12,-}=-k \delta_{k, l}=L_{21,-}, \\
L_{12,+}=\left(-k-2 \beta u_{k} v_{k}\right) \delta_{k, l}=L_{21,+} ;
\end{gathered}
$$

$L_{2,-}$ and $L_{2,+}$ can be obtained from Eqs. (A13) and (A14), through the exchange of $u$ and $v$ and of $\Lambda_{1}$ and $\Lambda_{2}$.

Now, using the equation for $U_{k} \quad\left(\omega U_{k}=L_{1,-} W_{k}\right.$ $+L_{12,-} Y_{k}$ ), multiplying it by $\omega$, and using the equations for $\omega W_{k}$ and $\omega Y_{k}$, we obtain a final formula for the eigenvalues (after forming the inner product of the resulting equation with the vector $\left.\left\langle U_{k}\right|\right)$

$$
\begin{aligned}
\omega^{2}= & \frac{1}{\left\langle U_{k} \mid U_{k}\right\rangle}\left[\left\langle U_{k}\left|\left(L_{1,-} L_{1,+}+L_{12,-} L_{21,+}\right)\right| U_{k}\right\rangle\right. \\
& \left.+\left\langle U_{k}\left|\left(L_{1,-} L_{12,+}+L_{12,-} L_{2,+}\right)\right| X_{k}\right\rangle\right] .
\end{aligned}
$$

In reaching Eq. (A17), we have made no assumptions on the nature of eigenfunctions and eigenvalues. From here on, we will assume that we are close to a case in which we know the phase eigenvalue $\omega_{\mathrm{ph}}=0$ and the translational one $\omega_{\text {tr }}$, namely, we will be perturbing around the case $\beta=\kappa=0$, in which the components are decoupled. In this case, $U_{k}=X_{k}$ and $W_{k}=Y_{k}$, while $u_{k}=v_{k}$. To the leading order, for the corrected eigenvalues (i.e., the ones in the perturbed cases of interest), we will be using as eigenvectors in Eq. (A17) the unperturbed ones. This gives us the additional equations (see, e.g., Ref. [36])

$$
\begin{aligned}
& L_{1,-} U_{k}=\omega_{\text {unp }} W_{k}, \\
& L_{1,+} W_{k}=\omega_{\text {unp }} U_{k},
\end{aligned}
$$

and the corresponding ones for $X_{k}, Y_{k}$ and $L_{2, \pm} ; \omega_{\text {unp }}$ is the eigenvalue of the unperturbed problem. Furthermore, for the phase modes, we have $U_{k}=\partial u_{k} / \partial \Lambda_{1}$ and $W_{k}=u_{k}$.

On the basis of the above relations, we obtain the following conclusions from Eq. (A17), upon algebraic manipulations:

(1) In the case $\kappa \neq 0, \beta=0$, and $\Lambda_{1}=\Lambda_{2}$, as discussed before, one of the pairs of the phase eigenvalues at the origin of the spectral plane $\left(\omega_{r}, \omega_{i}\right)$ will bifurcate away from the origin and may (depending on the sign of $\kappa$ ) cause an instability. The phase-mode bifurcation is described by

$$
\begin{aligned}
\omega^{2}= & \frac{1}{\left\langle U_{k} \mid U_{k}\right\rangle}\left[\kappa^{2} \sum_{k} U_{k}^{2}+2 \kappa \sum_{k} U_{k} u_{k}-2 \kappa \sum_{k} U_{k}^{2} u_{k}^{2}\right] \\
= & \frac{1}{\sum_{k}\left(\frac{\partial u_{k}}{\partial \Lambda_{1}}\right)^{2}}\left\{\kappa^{2} \sum_{k}\left(\frac{\partial u_{k}}{\partial \Lambda_{1}}\right)^{2}\right. \\
& \left.-2 \kappa \sum_{k}\left[\left(\frac{\partial u_{k}^{2}}{2 \partial \Lambda_{1}}\right)^{2}-\frac{\partial u_{k}^{2}}{2 \partial \Lambda_{1}}\right]\right\} .
\end{aligned}
$$

In this case, the translational modes can also bifurcate, but they are less "dangerous" in the sense of causing instabilities, as they are located either in the gap or in the continuous spectrum (depending on the value of $h$ ), while the phase modes bifurcate from the origin and hence they can directly lead to instability. We note, however, for the sake of completeness that the bifurcation of the translational modes can be obtained from Eq. (A24) below, by setting $\beta=0$.

(2) In the case $\kappa=0, \beta \neq 0$, and $\Lambda_{1}=\Lambda_{2}$, the phase modes are at the origin, so the translational modes are the only potential discrete-spectrum source of instabilities. In this case, for the translational modes, it will be

$$
\omega^{2}=\frac{1}{\sum_{k} U_{k}^{2}}\left[\omega_{t}^{2} \sum_{k} U_{k}^{2}-2 \beta \omega_{t} \sum_{k} W_{k} U_{k} u_{k}^{2}-4 \beta \sum_{k} U_{k}^{2} u_{k}^{2}\right] .
$$

(3) When $\beta \neq 0$ (and $\beta$ is fixed), $\kappa=0$, and $\Lambda_{1}-\Lambda_{2}$ $=\epsilon$, the translational eigenvalues will be once again responsible for possible instabilities, and the corresponding equation for them is

$$
\begin{aligned}
\omega^{2}= & \omega_{1, t}^{2}+\frac{1}{\sum_{k} U_{k}^{2}}\left[2 \omega_{t} \epsilon \sum_{k} U_{k} W_{k}+2 \epsilon \sum u_{k}^{2} U_{k}^{2}\right. \\
& \left.-2 \beta \epsilon \sum_{k} u_{k}^{2} U_{k}^{2}\right]
\end{aligned}
$$

where $\omega_{1, t}$ is given by Eq. (A24).

(4) Finally, in the general case when $\beta \neq 0$ and $\kappa \neq 0$, both translational and phase modes are of interest and can lead to instabilities. In this case, it will be true for the translational modes that

$$
\begin{array}{r}
\omega^{2}=\omega_{t}^{2}+\frac{1}{\sum_{k} U_{k}^{2}}\left[-2 \kappa \omega_{t} \sum_{k} U_{k} W_{k}+\kappa^{2} \sum_{k} U_{k}^{2}+2 \kappa(\beta\right. \\
\left.-1) \sum_{k} U_{k}^{2} u_{k}^{2}-2 \beta \omega_{t} \sum_{k} U_{k} W_{k} u_{k}^{2}-4 \beta \sum_{k} U_{k} u_{k}^{4}\right],
\end{array}
$$

while for the phase modes, we obtain

$$
\omega^{2}=\omega_{\beta=0}^{2}+\frac{1}{\sum_{k}\left(\frac{\partial u_{k}}{\partial \Lambda_{1}}\right)^{2}}\left[2 \kappa \beta \sum_{k}\left(\frac{\partial u_{k}^{2}}{2 \partial \Lambda_{1}}\right)^{2}\right]
$$

where $\omega_{\beta=0}$ is given by Eq. (A21). 
[1] A. Scott, Phys. Rep. 217, 1 (1992); O.M. Braun and Y.S. Kivshar, ibid. 306, 1 (1998); S. Flash and C.R. Willis, ibid. 295, 181 (1998); P.G. Kevrekidis, K.Ø. Rasmussen, and A.R. Bishop, Int. J. Mod. Phys. B 15, 2833 (2001).

[2] A.S. Davydov, J. Theor. Biol. 38, 559 (1973); A.S. Davydov, Biology and Quantum Mechanics (Pergamon Press, Oxford, 1982); A.C. Scott and L. Macneil, Phys. Lett. 98A, 87 (1983).

[3] D.N. Christodoulides and R.I. Joseph, Opt. Lett. 13, 794 (1988).

[4] W.P. Su, J.R. Schrieffer, and A.J. Heeger, Phys. Rev. Lett. 42, 1698 (1979); A.J. Sievers and S. Takeno, ibid. 61, 970 (1988).

[5] A. Trombettoni and A. Smerzi, Phys. Rev. Lett. 86, 2353 (2001); F.Kh. Abdullaev et al., Phys. Rev. A 64, 043606 (2001).

[6] H.S. Eisenberg, Y. Silberberg, R. Morandotti, A.R. Boyd, and J.S. Aitchison, Phys. Rev. Lett. 81, 3383 (1998).

[7] R. Morandotti, U. Peschel, J.S. Aitchison, H.S. Eisenberg, and Y. Silberberg, Phys. Rev. Lett. 83, 2726 (1999).

[8] H.S. Eisenberg, R. Morandotti, Y. Silberberg, J.M. Arnold, G. Pennelli, and J.S. Aitchison, J. Opt. Soc. Am. B 19, 2938 (2002).

[9] H.S. Eisenberg, Y. Silberberg, R. Morandotti, and J.S. Aitchison, Phys. Rev. Lett. 85, 1863 (2000).

[10] T. Pertsch, T. Zentgraf, U. Peschel, A. Brauer, and F. Lederer, Phys. Rev. Lett. 88, 093901 (2002).

[11] M.J. Ablowitz and Z.H. Musslimani, Phys. Rev. Lett. 87, 254102 (2001); U. Peschel and F. Lederer, J. Opt. Soc. Am. B 19, 544 (2002).

[12] D.N. Christodoulides and E.D. Eugenieva, Phys. Rev. Lett. 87, 233901 (2001).

[13] J. Pouget, M. Remoissenet, and J.M. Tamga, Phys. Rev. B 47, 14866 (1993).

[14] P.G. Kevrekidis, K.Ø. Rasmussen, and A.R. Bishop, Phys. Rev. E 61, 2006 (2000).

[15] E. W Laedke, K.H. Spatschek, and S.K. Turitsyn, Phys. Rev. Lett. 73, 1055 (1994).

[16] V.K. Mezentsev, S.L. Musher, I.V. Ryzhenkova, and S.K. Turitsyn, JETP Lett. 60, 828 (1994).

[17] A.B. Aceves, G.G. Luther, C. De Angelis, A.M. Rubenchik, and S.K. Turitsyn, Phys. Rev. Lett. 75, 73 (1995).

[18] H.S. Eisenberg, R. Morandotti, Y. Silberberg, S. Bar-Ad, D. Ross, and J.S. Aitchison, Phys. Rev. Lett. 87, 043902 (2001).

[19] J. W. Fleischer, M. Segev, N. K. Efremidis, and D. N. Christodoulides, Nature (London) 422, 147 (2003).

[20] N.K. Efremidis, S. Sears, D.N. Christodoulides, J.W. Fleischer, and M. Segev, Phys. Rev. E 66, 046602 (2002).

[21] R.J. Ballagh, K. Burnett, and T.F. Scott, Phys. Rev. Lett. 78, 1607 (1997).

[22] P. Ohberg and S. Stenholm, Phys. Rev. A 59, 3890 (1999); J. Williams, R. Walser, J. Cooper, E. Cornell, and M. Holland, ibid. 59, R31 (1999).

[23] S.E. Hamann, D.L. Haycock, G. Klose, P.H. Pax, I.H. Deutsch, and P.S. Jessen, Phys. Rev. Lett. 80, 4149 (1998).

[24] S. Darmanyan, A. Kobyakov, E. Schmidt, and F. Lederer, Phys. Rev. E 57, 3520 (1998).

[25] A. Kobyakov, S. Darmanyan, F. Lederer, and E. Schmidt, Opt. Quantum Electron. 30, 795 (1998).

[26] D.N. Christodoulides and R.I. Joseph, Opt. Lett. 13, 53 (1988);
D.J. Kaup, B.A. Malomed, and R.S. Tasgal, Phys. Rev. E 48, 3049 (1993); M. Haelterman and A. Sheppard, ibid. 49, 3376 (1994).

[27] M. Mitchell, M. Segev, and D.N. Christodoulides, Phys. Rev. Lett. 80, 4657 (1998).

[28] Z.H. Musslimani, M. Segev, D.N. Christodoulides, and M. Soljačič, Phys. Rev. Lett. 84, 1164 (2000).

[29] J.J. Garcia-Ripoll, V.M. Perez-Garcia, E.A. Ostrovskaya, and Yu.S. Kivshar, Phys. Rev. Lett. 85, 82 (2000).

[30] T. Carmon, C. Anastassiou, S. Lan, D. Kip, Z.H. Musslimani, M. Segev, and D.N. Christodoulides, Opt. Lett. 25, 1113 (2000); W. Krolikowski, E.A. Ostrovskaya, C. Weilnau, M. Geisser, G. McCarthy, Yu.S. Kivshar, C. Denz, and B. LutherDavies, Phys. Rev. Lett. 85, 1424 (2000).

[31] G.P. Agrawal, Applications of Nonlinear Fiber Optics (Academic, San Diego, 2001).

[32] D.N. Christodoulides and R.I. Joseph, Opt. Lett. 16, 446 (1991).

[33] S. Trillo, S. Wabnitz, W.C. Banyai, N. Finlayson, C.T. Seaton, G.I. Stegeman, and R.H. Stolen, Int. J. Quantum Chem. 25, 104 (1989).

[34] A.B. Aceves, C. De Angelis, and S. Wabnitz, Opt. Lett. 17, 1566 (1992).

[35] J.C. Eilbeck, P.S. Lomdahl, and A.C. Scott, Physica D 16, 318 (1985); J. Carr and J.C. Eilbeck, Phys. Lett. A 109, 201 (1985).

[36] M. Johansson and S. Aubry, Phys. Rev. E 61, 5864 (2000).

[37] P.G. Kevrekidis, K.Ø. Rasmussen, and A.R. Bishop, Phys. Rev. E 61, 2006 (2000).

[38] P.G. Kevrekidis, K.Ø. Rasmussen, and A.R. Bishop, Math. Comput. Simul. 55, 449 (2001).

[39] V.I. Arnold, Mathematical Aspects of Classical and Celestial Mechanics (Springer-Verlag, New York, 1997).

[40] T. Kapitula and P. Kevrekidis, Nonlinearity 14, 533 (2001).

[41] R. Boesch, P. Stancioff, and C.R. Willis, Phys. Rev. B 38, 6713 (1988).

[42] P.G. Kevrekidis, C.K.R.T. Jones, and T. Kapitula, Phys. Lett. A 269, 120 (2000)

[43] P.G. Kevrekidis, I.G. Kevrekidis, and A.R. Bishop, Phys. Lett. A 279, 361 (2001).

[44] B.A. Malomed, I.M. Skinner, P.L. Chu, and G.D. Peng, Phys. Rev. E 53, 4084 (1996).

[45] Y. Li and K. Promislow, Physica D 124, 137 (1998); Y. Li and K. Promislow, SIAM (Soc. Ind. Appl. Math.) J. Math. Anal. 31, 1351 (2000).

[46] M. Johansson and Yu.S. Kivshar, Phys. Rev. Lett. 82, 85 (1999).

[47] A.M. Morgante, M. Johansson, G. Kopidakis, and S. Aubry, Phys. Rev. Lett. 85, 550 (2000).

[48] P.G. Kevrekidis, A.R. Bishop, and K.Ø. Rasmussen, Phys. Rev. E 63, 036603 (2001).

[49] J.-C. van der Meer, Nonlinearity 3, 1041 (1990).

[50] P.G. Kevrekidis and T. Kapitula (unpublished).

[51] M. Grillakis, J. Shatah, and W. Strauss, J. Funct. Anal. 74, 160 (1987); M. Grillakis, Commun. Pure Appl. Math. 41, 745 (1988); 43, 299 (1990).

[52] T. Kapitula, P.G. Kevrekidis, and B.A. Malomed, Phys. Rev. E 63, 036604 (2001).

[53] D.J. Kaup, B.A. Malomed, and R.S. Tasgal, Phys. Rev. E 48, 
3049 (1993); B.A. Malomed and R.S. Tasgal, ibid. 58, 2564 (1998).

[54] D.N. Christodoulides and R.I. Joseph, Phys. Rev. Lett. 62, 1746 (1989); A. Aceves and S. Wabnitz, Phys. Lett. A 141, 37 (1989).

[55] C.M. de Sterke and J.E. Sipe, Prog. Opt. 33, 203 (1994).

[56] B.A. Malomed and R.S. Tasgal, Phys. Rev. E 49, 5787 (1994).

[57] I.V. Barashenkov, D.E. Pelinovsky, and E.V. Zemlyanaya,
Phys. Rev. Lett. 80, 5117 (1998).

[58] E.A. Ostrovskaya, Yu.S. Kivshar, D.V. Skryabin, and W.J. Firth, Phys. Rev. Lett. 83, 296 (1999).

[59] M. Johansson, S. Aubry, Yu.B. Gaididei, P.L. Christiansen, and K.Ø. Rasmussen, Physica D 119, 115 (1998).

[60] B.A. Malomed and P.G. Kevrekidis, Phys. Rev. E 64, 026601 (2001). 